\title{
Minkowski's inequality for the AB-fractional integral operator
}

\author{
Hasib Khan ${ }^{1,2 *}$, Thabet Abdeljawad ${ }^{3}$, Cemil Tunç $^{4}$, Abdulwasea Alkhazzan $^{5}$ and Aziz Khan ${ }^{6}$
}

"Correspondence:

hasibkhan13@yahoo.com

${ }^{1}$ College of Engineering Mechanics

and Materials, Hohai University,

Nanjing, P.R. China

${ }^{2}$ Department of Mathematics,

Shaheed BB University, Khybar

Pakhtunkhwa, Pakistan

Full list of author information is

available at the end of the article

\begin{abstract}
Recently, AB-fractional calculus has been introduced by Atangana and Baleanu and attracted a large number of scientists in different scientific fields for the exploration of diverse topics. An interesting aspect is the generalization of classical inequalities via AB-fractional integral operators. In this paper, we aim to generalize Minkowski inequality using the AB-fractional integral operator.
\end{abstract}

Keywords: AB-fractional integral operator; Minkowski inequality

\section{Introduction}

Nowadays the fractional calculus has an important role in diverse scientific fields due to its several applications in dynamical problems including signals, hydrodynamics, dynamics, fluid, viscoelastic theory, biology, control theory, image processing, computer networking, and many others [1-5]. A large number of scientists have worked on generalizations of existing results including theorems, definitions, models, and many more. A generalization of classical inequalities by means of fractional-order integral operators is considered as an interesting subject area. For instance, recently, Agarwal et al. [6] proved HermiteHadamard-type inequalities by using generalized $k$-fractional-integrals. Aldhaifallah et al. [7] used the $(k, s)$-fractional integral operator to generalize the inequalities for a family/class of $n$ positive functions. Set et al. [8] studied Hermite-Hadamard-type inequalities for a generalized fractional integral operator for functions with convex absolute values of derivatives. Khan et al. [9] produced the Minkowski inequality by using the Hahn integral operator. On the other hand, noninteger-order calculus, usually referred to as fractional calculus, is used to generalize integrals and derivatives, in particular, integrals involving inequalities. Recently, Dumitru and Arran [10] introduced a new formula for fractional derivatives and integrals by using the Mittag-Leffler kernel. More theoretical concepts regarding fractional operators with Mittag-Leffler kernels (Atangana-Baleanu operators) and the higher-order case have been discussed in $[11,12]$, whereas the generalization to the generalized Mittag-Leffler kernels to gain a semigroup property have been recently initiated in [13,14]. Khan [15] studied inequalities for a class of $n$ functions by means of Saigo fractional calculus. Jarad et al. [16] presented a Gronwall-type inequality for the analysis of the fractional-order Atangana-Baleanu differential equation and in [17] for generalized fractional derivatives.

(c) The Author(s) 2019. This article is distributed under the terms of the Creative Commons Attribution 4.0 International License (http://creativecommons.org/licenses/by/4.0/), which permits unrestricted use, distribution, and reproduction in any medium, provided you give appropriate credit to the original author(s) and the source, provide a link to the Creative Commons license, and indicate if changes were made. 
Shuang and Qi [18] proved some Hermite-Hadamard-type inequalities for a class of s-convex functions and studied special means. Mehrez and Agarwal [19] produced new integral inequalities by means of classical Hermite-Hadamard inequalities and obtained particular cases of their results with applications to special means. Park et al. [20] investigated new generalized inequalities, which then were utilized for stability analysis. Sarikaya et al. [21] established fractional integral inequalities generalizing the classical results by using the local fractional approach.

The integral inequalities with Mittag-Leffler functions have been studied as a generalization of the classical inequalities. For instance, Farid et al. [22] generalized several classical inequalities using an extended Mittag-Leffler function and evaluated particular cases of their results. More related work can be found in [23-25].

In this paper, we use the $\mathrm{AB}$-fractional integral operator for generalization of classical Minkowski inequalities. Our results are more general and applicable than those in the classical case. There are many definitions of fractional integrals, for example, RiemannLiouville, Hadamard, Liouville, Weyl, Erdelyi-Kober, and Katugampola [26-29], which can be considered for getting the same results. Now we give some definitions and lemma related to the AB-fractional operator.

Definition 1.1 ([30]) The fractional ABC-derivative in the Caputo sense of a function $f \in H^{*}(a, b)$ is defined by

$$
{ }_{A B C} \mathcal{D}_{\tau}^{v} f(\tau)=\frac{\mathbb{B}(v)}{1-v} \int_{a}^{\tau} f^{\prime}(s) E_{v}\left[\frac{-v(\tau-s)^{\mu}}{1-v}\right] d s,
$$

where $b>a$ and $v \in[0,1]$, and $\mathbb{B}(v)>0$ satisfies the property $\mathbb{B}(0)=\mathbb{B}(1)=1$.

Definition 1.2 The fractional ABC-derivative in the Riemann-Liouville sense of a function $f \in H^{*}(a, b)$ is defined by

$$
{ }_{a}^{A B R} \mathcal{D}_{\tau}^{v} f(\tau)=\frac{\mathbb{B}(v)}{1-v} \frac{d}{d \tau} \int_{a}^{\tau} f(s) E_{v}\left[\frac{-v(\tau-s)^{v}}{1-v}\right] d s,
$$

where $b>a$ and $v \in[0,1]$.

Definition $1.3([31,32])$ The fractional AB-integral of the function $f \in H^{*}(a, b)$ is given by

$$
{ }_{a}^{A B} \mathcal{I}_{\tau}^{v} f(\tau)=\frac{1-v}{\mathbb{B}(\nu)} f(\tau)+\frac{v}{\mathbb{B}(v) \Gamma(v)} \int_{a}^{\tau} f(s)(\tau-s)^{\nu-1} d s,
$$

where $b>a$ and $0<v<1$.

Remark 1.4 Since the normalization function $\mathbb{B}(v)>0$ is positive, it immediately follows that the AB-integral of a positive function is positive. We will rely on this fact throughout the proofs of the main results.

Lemma 1.5 ([33]) The ABC-fractional derivative and AB-fractional integral of a function $f$ satisfy the Newton-Leibnitz formula

$$
{ }_{a}^{A B} \mathcal{I}_{\tau}^{v}\left({ }_{a}^{A B C} \mathcal{D}_{\tau}^{v} f(\tau)\right)=f(\tau)-f(a) .
$$


Organization of the paper. This paper includes four sections. Introduction is given in Sect. 1, with a literature review, important definitions, and a lemma, which we will use in the proofs. In Sect. 2, we prove Minkowski's inequality for the AB-fractional integral operator. Other AB-fractional integral inequalities are proved in Sect. 3. The summary is given in Sect. 4.

\section{The AB-fractional Minkowski inequality}

Theorem 2.1 Let $v>0$ and $p \geq 1$. Let $u, v \in C_{v}[a, b]$ be two positive functions in $[0, \infty[$ such that ${ }_{a}^{A B} \mathcal{I}_{t}^{v} u(t)<\infty$ and ${ }^{A B}{ }_{a} \mathcal{I}_{t}^{v} v(t)<\infty$ for all $t>a$. If $0<\alpha \leq \frac{u(t)}{v(t)} \leq \theta$ for some $\alpha, \theta \in \mathbb{R}_{+}^{*}$ and all $t \in[a, b]$, then

$$
\left({ }^{A B}{ }_{a} \mathcal{I}_{t}^{v} u^{p}(t)\right)^{\frac{1}{p}}+\left({ }^{A B}{ }_{a} \mathcal{I}_{t}^{v} v^{p}(t)\right)^{\frac{1}{p}} \leq \mathcal{A}\left[{ }^{A B}{ }_{a} \mathcal{I}_{t}^{v}(u(t)+v(t))^{p}\right]^{\frac{1}{p}}
$$

where

$$
\mathcal{A}=\frac{\theta(1+\alpha)+(\theta+1)}{(1+\alpha)(\theta+1)} \text {. }
$$

Proof From the condition $\frac{u(t)}{v(t)} \leq \theta$ we obtain

$$
u(t) \leq\left(\frac{\theta}{\theta+1}\right)(u(t)+v(t))
$$

Taking the $p$ th power of both sides of Eq. (2.2), we have

$$
u^{p}(t) \leq\left(\frac{\theta}{\theta+1}\right)^{p}(u(t)+v(t))^{p}
$$

Multiplying both sides of (2.3) by $\frac{1-v}{\mathbb{B}(v)}$, we get

$$
\frac{1-v}{\mathbb{B}(v)} u^{p}(t) \leq\left(\frac{\theta}{\theta+1}\right)^{p} \frac{1-v}{\mathbb{B}(v)}(u(t)+v(t))^{p} .
$$

Also, replacing $t$ by $s$ in Eq. (2.3) and multiplying both sides by $\frac{v(t-s)^{v-1}}{\mathbb{B}(v) \Gamma(v)}$, we get

$$
\frac{v(t-s)^{\nu-1}}{\mathbb{B}(\nu) \Gamma(v)} u^{p}(s) \leq\left(\frac{\theta}{\theta+1}\right)^{p} \frac{v(t-s)^{\nu-1}}{\mathbb{B}(v) \Gamma(v)}(u(s)+v(s))^{p} .
$$

Integrating both sides of Eq. (2.4) with respect to $s$, we have

$$
\int_{a}^{t} \frac{v(t-s)^{\nu-1}}{\mathbb{B}(v) \Gamma(v)} u^{p}(s) d s \leq\left(\frac{\theta}{\theta+1}\right)^{p} \int_{a}^{t} \frac{v(t-s)^{\nu-1}}{\mathbb{B}(v) \Gamma(v)}(u(s)+v(s))^{p} d s .
$$

Adding (2.4) and (2.6), we obtain

$$
\begin{aligned}
\frac{1-v}{\mathbb{B}(v)} u^{p}(t)+\int_{a}^{t} \frac{v(t-s)^{v-1}}{\mathbb{B}(v) \Gamma(v)} u^{p}(s) d s \leq & \left(\frac{\theta}{\theta+1}\right)^{p}\left[\frac{1-v}{\mathbb{B}(v)}(u(t)+v(t))^{p}\right. \\
& \left.+\int_{a}^{t} \frac{v(t-s)^{\nu-1}}{\mathbb{B}(v) \Gamma(v)}(u(s)+v(s))^{p} d s\right] .
\end{aligned}
$$


This implies

$$
{ }_{a}^{A B} \mathcal{I}_{t}^{v} u^{p}(s) \leq\left(\frac{\theta}{\theta+1}\right)^{p}{ }_{a} \mathcal{I}_{t}^{v}(u(s)+v(s))^{p} .
$$

Taking the $\frac{1}{p}$ th power of both sides of Eq. (2.7), we find

$$
\left({ }^{A B} \mathcal{I}_{t}^{v} u^{p}(t)\right)^{\frac{1}{p}} \leq \frac{\theta}{\theta+1}\left[{ }^{A B}{ }_{a} \mathcal{I}_{t}^{v}(u(t)+v(t))^{p}\right]^{\frac{1}{p}}
$$

On the other hand, by using the condition $0<\alpha \leq \frac{u(t)}{v(t)}$ we directly get

$$
v^{p}(t) \leq \frac{1}{(1+\alpha)^{p}}(u(t)+v(t))^{p}
$$

Multiplying Eq. (2.9) by $\frac{1-v}{\mathbb{B}(v)}$, we get

$$
\frac{1-v}{\mathbb{B}(v)} v^{p}(t) \leq \frac{1}{(1+\alpha)^{p}} \frac{1-v}{\mathbb{B}(v)}(u(t)+v(t))^{p} .
$$

Also, replacing $t$ by $s$ in Eq. (2.9) and multiplying both sides by $\frac{v(t-s)^{\nu-1}}{\mathbb{B}(v) \Gamma(v)}$, we get

$$
\frac{\nu(t-s)^{\nu-1}}{\mathbb{B}(\nu) \Gamma(\nu)} v^{p}(s) \leq \frac{1}{(1+\alpha)^{p}} \frac{v(t-s)^{\nu-1}}{\mathbb{B}(\nu) \Gamma(\nu)}(u(s)+v(s))^{p} .
$$

Integrating both sides of Eq. (2.11) with respect to $s$, we have

$$
\int_{a}^{t} \frac{v(t-s)^{\nu-1}}{\mathbb{B}(v) \Gamma(v)} v^{p}(s) d s \leq \frac{1}{(1+\alpha)^{p}} \int_{a}^{t} \frac{v(t-s)^{\nu-1}}{\mathbb{B}(v) \Gamma(v)}(u(s)+v(s))^{p} d s .
$$

Adding (2.10) and (2.12), we obtain

$$
\begin{aligned}
\frac{1-v}{\mathbb{B}(v)} v^{p}(t)+\int_{a}^{t} \frac{v(t-s)^{\nu-1}}{\mathbb{B}(v) \Gamma(v)} v^{p}(s) d s \leq & \frac{1}{(1+\alpha)^{p}} \frac{1-v}{\mathbb{B}(v)}(u(t)+v(t))^{p} \\
& \left.+\int_{a}^{t} \frac{v(t-s)^{v-1}}{\mathbb{B}(v) \Gamma(v)}(u(s)+v(s))^{p} d s\right] .
\end{aligned}
$$

This leads to the AB-fractional integral inequality

$$
{ }_{a}^{A B} \mathcal{I}_{t}^{v} v^{p}(t) \leq \frac{1}{(1+\alpha)^{p}}{ }_{a}^{A B} \mathcal{I}_{t}^{v}(u(t)+v(t))^{p}
$$

Taking the $\frac{1}{p}$ th power of both sides of Eq. (2.14), we find

$$
\left({ }^{A B}{ }_{a} \mathcal{I}_{t}^{v} v^{p}(t)\right)^{\frac{1}{p}} \leq \frac{1}{1+\alpha}\left[{ }_{a}^{A B} \mathcal{I}_{t}^{v}(u(t)+v(t))^{p}\right]^{\frac{1}{p}}
$$

By Eqs. (2.8) and (2.15) we obtain

$$
\left({ }^{A B}{ }_{a} \mathcal{I}_{t}^{v} u^{p}(t)\right)^{\frac{1}{p}}+\left({ }^{A B}{ }_{a} \mathcal{I}_{t}^{v} v^{p}(t)\right)^{\frac{1}{p}} \leq \mathcal{A}\left[{ }^{A B}{ }_{a} \mathcal{I}_{t}^{v}(u(t)+v(t))^{p}\right]^{\frac{1}{p}}
$$

Thus, the proof of the AB-fractional integral inequality is completed. 


\section{Other types of inequalities}

Theorem 3.1 Let $v>0$ and $p>1, q>1, \frac{1}{p}+\frac{1}{q}=1$. Let $u, v \in C_{v}[a, b]$ be two positive functions in $\left[0, \infty\left[\right.\right.$ such that ${ }_{a}^{A B} \mathcal{I}_{t}^{v} u(t)<\infty$ and ${ }^{A B}{ }_{a} \mathcal{I}_{t}^{v} v(t)<\infty$ for all $t>a$. If $0<\alpha \leq \frac{u(t)}{v(t)} \leq \theta$ for some $\alpha, \theta \in \mathbb{R}_{+}^{*}$ and all $t \in[a, b]$, then

$$
\left({ }_{a}^{A B} \mathcal{I}_{t}^{v} u(t)\right)^{\frac{1}{p}}\left({ }_{a}^{A B} \mathcal{I}_{t}^{v} v(t)\right)^{\frac{1}{q}} \leq\left(\frac{\theta}{\alpha}\right)^{\frac{1}{p q}}\left[{ }_{a}^{A B} \mathcal{I}_{t}^{v}\left(u^{\frac{1}{p}}(t) v^{\frac{1}{q}}(t)\right)\right]
$$

Proof Using the condition $\frac{u(t)}{v(t)} \leq \theta$, we get

$$
u^{\frac{1}{q}} \leq \theta^{\frac{1}{q}} v^{\frac{1}{q}}
$$

Multiplying (3.2) by $u^{\frac{1}{p}}$ and using the condition $\frac{1}{p}+\frac{1}{q}=1$, we have

$$
u \leq \theta^{\frac{1}{q}} u^{\frac{1}{p}} v^{\frac{1}{q}}
$$

Now let us use (3.3) twice. First, multiplying by $\frac{1-v}{\mathbb{B}(v)}$, we get

$$
\frac{1-v}{\mathbb{B}(v)} u \leq \theta^{\frac{1}{q}} \frac{1-v}{\mathbb{B}(v)} u^{\frac{1}{p}} v^{\frac{1}{q}} .
$$

Second, multiplying by $\frac{v(t-s)^{v-1}}{\mathbb{B}(v) \Gamma(v)}$, we obtain

$$
\frac{v(t-s)^{\nu-1}}{\mathbb{B}(v) \Gamma(v)} u \leq \theta^{\frac{1}{q}} \frac{v(t-s)^{v-1}}{\mathbb{B}(v) \Gamma(v)} u^{\frac{1}{p}} v^{\frac{1}{q}} .
$$

Integrating both sides of Eq. (3.5) from 0 to $t$, we have

$$
\int_{a}^{t} \frac{v(t-s)^{\nu-1}}{\mathbb{B}(v) \Gamma(v)} u(s) d s \leq \theta^{\frac{1}{q}} \int_{a}^{t} \frac{v(t-s)^{\nu-1}}{\mathbb{B}(v) \Gamma(v)} u(s)^{\frac{1}{p}} v(s)^{\frac{1}{q}} d s .
$$

Now, by adding Eq. (3.4) and Eq. (3.6) we find

$$
\begin{aligned}
\frac{1-v}{\mathbb{B}(v)} u(t)+\int_{a}^{t} \frac{v(t-s)^{\nu-1}}{\mathbb{B}(v) \Gamma(v)} u(s) d s \leq & \theta^{\frac{1}{q}}\left[\frac{1-v}{\mathbb{B}(v)} u(t)^{\frac{1}{p}} v(t)^{\frac{1}{q}}\right. \\
& \left.+\int_{a}^{t} \frac{v(t-s)^{v-1}}{\mathbb{B}(v) \Gamma(v)} u(s)^{\frac{1}{p}} v(s)^{\frac{1}{q}} d s\right] .
\end{aligned}
$$

This implies

$$
{ }_{a}^{A B} \mathcal{I}_{t}^{v} u(t) \leq \theta^{\frac{1}{q}}\left[{ }_{a}^{A B} \mathcal{I}_{t}^{\mathcal{v}}\left(u^{\frac{1}{p}}(t) v^{\frac{1}{q}}(t)\right)\right]
$$

Taking the $\frac{1}{p}$ th power of both sides of (3.7), we have

$$
\left[{ }_{a}^{A B} \mathcal{I}_{t}^{v} u(t)\right]^{\frac{1}{p}} \leq \theta^{\frac{1}{p q}}\left[{ }_{a}^{A B} \mathcal{I}_{t}^{v}\left(u^{\frac{1}{p}}(t) v^{\frac{1}{q}}(t)\right)\right]^{\frac{1}{p}}
$$


Now, by the condition $\alpha \leq \frac{u(t)}{v(t)}$ we have

$$
v^{\frac{1}{p}} \leq \alpha^{\frac{-1}{p}} u^{\frac{1}{p}}
$$

Multiplying Eq. (3.9) by $v^{\frac{1}{q}}$, we get

$$
v \leq \alpha^{\frac{-1}{p}} u^{\frac{1}{p}} v^{\frac{1}{q}}
$$

Now let us use (3.10) twice. First, multiplying by $\frac{1-v}{\mathbb{B}(v)}$, we get

$$
\frac{1-v}{\mathbb{B}(v)} v \leq \alpha^{\frac{-1}{p}} \frac{1-v}{\mathbb{B}(v)} u^{\frac{1}{p}} v^{\frac{1}{q}}
$$

Second, multiplying by $\frac{v(t-s)^{v-1}}{\mathbb{B}(v) \Gamma(v)}$, we obtain

$$
\frac{\nu(t-s)^{\nu-1}}{\mathbb{B}(\nu) \Gamma(\nu)} v \leq \alpha^{\frac{-1}{p}} \frac{v(t-s)^{\nu-1}}{\mathbb{B}(\nu) \Gamma(\nu)} u^{\frac{1}{p}} v^{\frac{1}{q}}
$$

Integrating both sides of Eq. (3.12) from 0 to $t$, we have

$$
\int_{a}^{t} \frac{v(t-s)^{\nu-1}}{\mathbb{B}(v) \Gamma(v)} v(s) d s \leq \alpha^{\frac{-1}{p}} \int_{a}^{t} \frac{v(t-s)^{\nu-1}}{\mathbb{B}(v) \Gamma(v)} u(s)^{\frac{1}{p}} \nu(s)^{\frac{1}{q}} d s .
$$

Now, by adding Eq. (3.11) and Eq. (3.13) we find

$$
\begin{aligned}
\frac{1-v}{\mathbb{B}(v)} v(t)+\int_{a}^{t} \frac{v(t-s)^{v-1}}{\mathbb{B}(v) \Gamma(v)} v(s) d s \leq & \alpha^{\frac{-1}{p}}\left[\frac{1-v}{\mathbb{B}(v)} u(t)^{\frac{1}{p}} v(t)^{\frac{1}{q}}\right. \\
& \left.+\int_{a}^{t} \frac{v(t-s)^{v-1}}{\mathbb{B}(v) \Gamma(v)} u(s)^{\frac{1}{p}} v(s)^{\frac{1}{q}} d s\right] .
\end{aligned}
$$

This implies

$$
{ }_{a}^{A B} \mathcal{I}_{t}^{v} v(t) \leq \alpha^{\frac{-1}{p}}\left[{ }^{A B} \mathcal{I}_{t}^{v}\left(u^{\frac{1}{p}}(t) v^{\frac{1}{q}}(t)\right)\right]
$$

Taking the $\frac{1}{q}$ th power of both sides of (3.15), we have

$$
\left[{ }^{A B}{ }_{a} \mathcal{I}_{t}^{v} v(s)\right]^{\frac{1}{q}} \leq \alpha^{\frac{-1}{p q}}\left[{ }_{a}^{A B} \mathcal{I}_{t}^{v}\left(u^{\frac{1}{p}}(t) v^{\frac{1}{q}}(t)\right)\right]^{\frac{1}{q}}
$$

Finally, multiplying Eq. (3.8) and Eq. (3.16), we obtain

$$
\left({ }_{a}^{A B} \mathcal{I}_{t}^{v} u(t)\right)^{\frac{1}{p}}\left({ }^{A B}{ }_{a} \mathcal{I}_{t}^{v} v(t)\right)^{\frac{1}{q}} \leq\left(\frac{\theta}{\alpha}\right)^{\frac{1}{p q}}\left[{ }^{A B}{ }_{a} \mathcal{I}_{t}^{v}\left(u^{\frac{1}{p}}(t) v^{\frac{1}{q}}(t)\right)\right]
$$

Theorem 3.2 Let $v>0$ and $p>1, q>1, \frac{1}{p}+\frac{1}{q}=1$. Let $u, v \in C_{v}[a, b]$ be two positive functions in $\left[0, \infty\left[\right.\right.$ such that ${ }^{A B}{ }_{a} \mathcal{I}_{t}^{v} u^{p}(t)<\infty,{ }_{a}{ }_{a} \mathcal{I}_{t}^{v} u^{q}(t)<\infty,{ }^{A B}{ }_{a} \mathcal{I}_{t}^{v} v^{p}(t)<\infty$, and ${ }_{a B} \mathcal{I}_{t}^{v} \nu^{q}(t)<\infty$ for all $t>a$. If $0<\alpha \leq \frac{u(t)}{v(t)} \leq \theta$ for some $\alpha, \theta \in \mathbb{R}_{+}^{*}$ and all $t \in[a, b]$, then

$$
{ }_{a}^{A B} \mathcal{I}_{t}^{v}(u(t)+v(t)) \leq \mathcal{A}^{* A B}{ }_{a} \mathcal{I}_{t}^{v}\left(u^{p}(t)+v^{p}(t)\right)+\mathcal{B}_{m}^{* A B}{ }_{a} \mathcal{I}_{t}^{v}\left(u^{q}(t)+v^{q}(t)\right)
$$


where

$$
\mathcal{A}^{*}=\frac{2^{p-1} \theta^{p}}{p(\theta+1)^{p}}, \quad \mathcal{B}_{m}^{*}=\frac{2^{q-1}}{q(1+\alpha)^{q}} .
$$

Proof Using the condition $\frac{u(t)}{v(t)} \leq \theta$, we obtain

$$
u(t) \leq\left(\frac{\theta(u(t)+v(t))}{1+\theta}\right) .
$$

Taking the $p$ th power of both sides of Eq. (2.2), we have

$$
u^{p}(t) \leq\left(\frac{\theta}{\theta+1}\right)^{p}(u(t)+v(t))^{p} .
$$

Multiplying both sides of (3.20) by $\frac{1-v}{\mathbb{B}(v)}$, we get

$$
\frac{1-v}{\mathbb{B}(v)} u^{p}(t) \leq\left(\frac{\theta}{\theta+1}\right)^{p} \frac{1-v}{\mathbb{B}(v)}(u(t)+v(t))^{p} .
$$

Also, replacing $t$ by $s$ in Eq. (3.20) and multiplying both sides by $\frac{v(t-s)^{\nu-1}}{\mathbb{B}(v) \Gamma(v)}$, we get

$$
\frac{v(t-s)^{\nu-1}}{\mathbb{B}(v) \Gamma(v)} u^{p}(s) \leq\left(\frac{\theta}{\theta+1}\right)^{p} \frac{v(t-s)^{\nu-1}}{\mathbb{B}(v) \Gamma(v)}(u(s)+v(s))^{p} .
$$

Integrating both sides of Eq. (3.21) with respect to $s$, we have

$$
\int_{a}^{t} \frac{v(t-s)^{\nu-1}}{\mathbb{B}(v) \Gamma(v)} u^{p}(s) d s \leq\left(\frac{\theta}{\theta+1}\right)^{p} \int_{a}^{t} \frac{v(t-s)^{\nu-1}}{\mathbb{B}(v) \Gamma(v)}(u(s)+v(s))^{p} d s .
$$

Adding (3.21) and (3.23), we obtain

$$
\begin{aligned}
& \frac{1-v}{\mathbb{B}(v)} u^{p}(t)+\int_{a}^{t} \frac{v(t-s)^{v-1}}{\mathbb{B}(v) \Gamma(v)} u^{p}(s) d s \\
& \quad \leq\left(\frac{\theta}{\theta+1}\right)^{p}\left[\frac{1-v}{\mathbb{B}(v)}(u(t)+v(t))^{p}+\int_{a}^{t} \frac{v(t-s)^{v-1}}{\mathbb{B}(v) \Gamma(v)}(u(s)+v(s))^{p} d s\right] .
\end{aligned}
$$

This implies

$$
{ }_{a B} \mathcal{I}_{t}^{v} u^{p}(t) \leq\left(\frac{\theta}{\theta+1}\right)^{p}{ }_{a B} \mathcal{I}_{t}^{v}(u(t)+v(t))^{p}
$$

Multiplying (2.7) by the constant $\frac{1}{p}$, we find

$$
\frac{1}{p}\left({ }_{a}^{A B} \mathcal{I}_{t}^{v} u^{p}(t)\right) \leq \frac{1}{p}\left(\frac{\theta}{\theta+1}\right)^{p}\left[{ }_{a}^{A B} \mathcal{I}_{t}^{v}(u(t)+v(t))^{p}\right] .
$$

On the other hand, by using the condition $0<\alpha \leq \frac{u(t)}{v(t)}$ we directly get

$$
v^{q}(t) \leq \frac{1}{(1+\alpha)^{q}}(u(t)+v(t))^{q} .
$$


Multiplying (3.26) by $\frac{1-v}{\mathbb{B}(v)}$, we get

$$
\frac{1-v}{\mathbb{B}(v)} v^{q}(t) \leq \frac{1}{(1+\alpha)^{q}} \frac{1-v}{\mathbb{B}(v)}(u(t)+v(t))^{q} .
$$

Also, replacing $t$ by $s$ in Eq. (3.26) and multiplying both sides by $\frac{v(t-s)^{v-1}}{\mathbb{B}(v) \Gamma(v)}$, we get

$$
\frac{v(t-s)^{\nu-1}}{\mathbb{B}(v) \Gamma(v)} v^{q}(s) \leq \frac{1}{(1+\alpha)^{q}} \frac{v(t-s)^{\nu-1}}{\mathbb{B}(v) \Gamma(v)}(u(s)+v(s))^{q}
$$

Integrating both sides of Eq. (3.28) with respect to $s$, we have

$$
\int_{a}^{t} \frac{v(t-s)^{v-1}}{\mathbb{B}(v) \Gamma(v)} v^{q}(s) d s \leq \frac{1}{(1+\alpha)^{q}} \int_{a}^{t} \frac{v(t-s)^{v-1}}{\mathbb{B}(v) \Gamma(v)}(u(s)+v(s))^{q} d s
$$

Adding (3.27) and (3.29), we obtain

$$
\begin{aligned}
\frac{1-v}{\mathbb{B}(v)} v^{q}(t)+\int_{a}^{t} \frac{v(t-s)^{v-1}}{\mathbb{B}(v) \Gamma(v)} v^{q}(s) d s \leq & \frac{1}{(1+\alpha)^{q}}\left[\frac{1-v}{\mathbb{B}(v)}(u(t)+v(t))^{q}\right. \\
& \left.+\int_{a}^{t} \frac{v(t-s)^{\nu-1}}{\mathbb{B}(v) \Gamma(v)}(u(s)+v(s))^{q} d s\right] .
\end{aligned}
$$

This implies

$$
{ }_{a}^{A B} \mathcal{I}_{t}^{v} v^{q}(t) \leq \frac{1}{(1+\alpha)^{q}}{ }_{a}^{A B} \mathcal{I}_{t}^{v}(u(t)+v(t))^{q} .
$$

Multiplying (2.14) by $\frac{1}{q}$, we have

$$
\frac{1}{q}\left({ }^{A B}{ }_{a} \mathcal{I}_{t}^{v} v^{q}(t)\right) \leq \frac{1}{q} \frac{1}{(1+\alpha)^{q}}\left[{ }^{A B}{ }_{a} \mathcal{I}_{t}^{v}(u(t)+v(t))^{q}\right]
$$

By means of Eqs. (3.25) and (3.31) we get

$$
\begin{aligned}
& \frac{1}{p}\left({ }^{A B}{ }_{a} \mathcal{I}_{t}^{v} u^{p}(t)\right)+\frac{1}{q}\left({ }^{A B}{ }_{a} \mathcal{I}_{t}^{v} v^{q}(t)\right) \\
& \quad \leq \frac{1}{p}\left(\frac{\theta}{\theta+1}\right)^{p}\left[{ }^{A B}{ }_{a} \mathcal{I}_{t}^{v}(u(t)+v(t))^{p}\right]+\frac{1}{q} \frac{1}{(1+\alpha)^{q}}\left[{ }^{A B}{ }_{a} \mathcal{I}_{t}^{v}(u(t)+v(t))^{q}\right] .
\end{aligned}
$$

To complete our proof, we have to use Young's inequality

$$
u(t) v(t) \leq \frac{u^{p}(t)}{p}+\frac{v^{q}(t)}{q}
$$

Multiplying (3.33) by $\frac{1-v}{\mathbb{B}(v)}$, we get

$$
\frac{1-v}{\mathbb{B}(v)} u(t) v(t) \leq \frac{1-v}{\mathbb{B}(v)}\left(\frac{u^{p}(t)}{p}+\frac{v^{q}(t)}{q}\right) .
$$


Khan et al. Journal of Inequalities and Applications

(2019) 2019:96

Page 9 of 12

Also, replacing $t$ by $s$ in Eq. (3.33) and multiplying both sides by $\frac{v(t-s)^{v-1}}{\mathbb{B}(v) \Gamma(v)}$, we get

$$
\frac{v(t-s)^{\nu-1}}{\mathbb{B}(v) \Gamma(v)} u(s) v(s) \leq \frac{v(t-s)^{\nu-1}}{p \mathbb{B}(v) \Gamma(v)} u^{p}(s)+\frac{v(t-s)^{\nu-1}}{q \mathbb{B}(v) \Gamma(v)} v^{q}(s) .
$$

Integrating both sides of Eq. (3.35) with respect to $s$, we have

$$
\int_{a}^{t} \frac{v(t-s)^{\nu-1}}{\mathbb{B}(v) \Gamma(v)} u(s) v(s) d s \leq \int_{a}^{t} \frac{v(t-s)^{\nu-1}}{p \mathbb{B}(v) \Gamma(v)} u^{p}(s) d s+\int_{a}^{t} \frac{v(t-s)^{\nu-1}}{q \mathbb{B}(v) \Gamma(v)} v^{q}(s) d s .
$$

Adding (3.34) and (3.36), we obtain

$$
\begin{aligned}
\frac{1-v}{\mathbb{B}(v)} u(t) v(t)+\int_{a}^{t} \frac{v(t-s)^{v-1}}{\mathbb{B}(v) \Gamma(v)} u(s) v(s) d s \\
\leq \leq \frac{1-v}{\mathbb{B}(v)}\left(\frac{u^{p}(t)}{p}+\frac{v^{q}(t)}{q}\right) \\
\quad+\int_{a}^{t} \frac{v(t-s)^{v-1}}{p \mathbb{B}(v) \Gamma(v)} u^{p}(s) d s+\int_{a}^{t} \frac{v(t-s)^{v-1}}{q \mathbb{B}(v) \Gamma(v)} v^{q}(s) d s .
\end{aligned}
$$

This implies

$$
{ }_{a B} \mathcal{I}_{t}^{v} u(t) v(t) \leq \frac{1}{p}{ }_{a B} \mathcal{I}_{t}^{v} u^{p}(t)+\frac{1}{q}{ }_{a B} \mathcal{I}_{t}^{v} v^{q}(t)
$$

Using (3.32) and (3.38), we have

$$
\begin{aligned}
& { }_{a}^{A B} \mathcal{I}_{t}^{v} u(t) v(t) \\
& \quad \leq \frac{1}{p}\left(\frac{\theta}{\theta+1}\right)^{p}\left[{ }_{a}^{A B} \mathcal{I}_{t}^{v}(u(t)+v(t))^{p}\right]+\frac{1}{q} \frac{1}{(1+\alpha)^{q}}\left[{ }_{a}^{A B} \mathcal{I}_{t}^{v}(u(t)+v(t))^{q}\right] .
\end{aligned}
$$

Using the inequality

$$
(u+v)^{r} \leq 2^{r-1}\left(u^{r}+v^{r}\right), \quad u, v \geq 0, r>1,
$$

with $r=p$ and multiplying (3.40) by the constant $\frac{1-v}{\mathbb{B}(v)}$, we find

$$
\frac{1-v}{\mathbb{B}(v)}(u(t)+v(t))^{p} \leq 2^{p-1} \frac{1-v}{\mathbb{B}(v)}\left(u(t)^{p}+v(t)^{p}\right) .
$$

Then multiplying Eq. (3.40) with $r=p$ by $\frac{v(t-s)^{\nu-1}}{\mathbb{B}(v) \Gamma(v)}$, we get

$$
\frac{v(t-s)^{\nu-1}}{\mathbb{B}(v) \Gamma(v)}(u+v)^{p} \leq 2^{p-1} \frac{v(t-s)^{v-1}}{\mathbb{B}(v) \Gamma(v)}\left(u^{p}+v^{p}\right) .
$$

Integrating Eq. (3.42) from $a$ to $t$, we have

$$
\int_{a}^{t} \frac{v(t-s)^{\nu-1}}{\mathbb{B}(v) \Gamma(v)}(u(s)+v(s))^{p} d s \leq 2^{p-1} \int_{a}^{t} \frac{v(t-s)^{\nu-1}}{\mathbb{B}(v) \Gamma(v)}\left(u^{p}(s)+v^{p}(s)\right) d s .
$$


Adding Eq. (3.41) and Eq. (3.43), we obtain

$$
\begin{aligned}
& \frac{1-v}{\mathbb{B}(v)}(u(t)+v(t))^{p}+\int_{a}^{t} \frac{v(t-s)^{v-1}}{\mathbb{B}(v) \Gamma(v)}(u(s)+v(s))^{p} d s \\
& \quad \leq 2^{p-1}\left(\frac{1-v}{\mathbb{B}(v)}\left(u(t)^{p}+v(t)^{p}\right)+\int_{a}^{t} \frac{v(t-s)^{v-1}}{\mathbb{B}(v) \Gamma(v)}\left(u^{p}(s)+v^{p}(s)\right) d s\right) .
\end{aligned}
$$

This implies

$$
{ }_{a}^{A B} \mathcal{I}_{t}^{v}(u(t)+v(t))^{p} \leq 2^{p-1 A B}{ }_{a} \mathcal{I}_{t}^{v}\left(u^{p}(t)+v^{p}(t)\right) .
$$

Repeating the same process with $r=q$, we get

$$
{ }_{a}^{A B} \mathcal{I}_{t}^{v}(u(t)+v(t))^{q} \leq 2^{q-1 A B}{ }_{a} \mathcal{I}_{t}^{v}\left(u^{q}(t)+v^{q}(t)\right) .
$$

Substituting by (3.45) and (3.46) into Eq. (3.39), the proof completed.

Theorem 3.3 Let $v>0$, and let $u, v \in C_{v}[a, b]$ be two positive functions in $[0, \infty$ [ such that ${ }_{a}^{A B} \mathcal{I}_{t}^{v} u(t)<\infty$ and ${ }^{A B}{ }_{a} \mathcal{I}_{t}^{v} v(t)<\infty$ for all $t>a$, If $0<\alpha \leq \frac{u(t)}{v(t)} \leq \theta$ for some $\alpha, \theta \in \mathbb{R}_{+}^{*}$ and all $t \in[a, b]$, then

$$
\frac{1_{A B}}{\theta}{ }_{a} \mathcal{I}_{t}^{v}(u(t) v(t)) \leq{ }^{A B}{ }_{a} \mathcal{I}_{t}^{v}(u(t)+v(t))^{2} \leq \frac{1}{\alpha}{ }_{a B} \mathcal{I}_{t}^{v}(u(t) v(t))
$$

Proof Using the condition

$$
0<\alpha \leq \frac{u(t)}{v(t)} \leq \theta
$$

we conclude that

$$
\begin{aligned}
& (1+\alpha) v(t) \leq(u(t)+v(t)) \leq(\theta+1) v(t), \\
& \frac{\theta+1}{\theta} u(t) \leq(u(t)+v(t)) \leq \frac{1+\alpha}{\alpha} u(t) .
\end{aligned}
$$

By (3.49) and (3.50) we obtain

$$
\frac{1}{\theta} u(t) v(t) \leq \frac{(u(t)+v(t))^{2}}{(1+\alpha)(\theta+1)} \leq \frac{1}{\alpha} u(t) v(t)
$$

Multiplying (3.51) by $\frac{1-v}{\mathbb{B}(v)}$ and then by $\frac{v(t-s)^{v-1}}{\mathbb{B}(v) \Gamma(v)}$, we get

$$
\begin{aligned}
& \frac{1}{\theta} \frac{1-v}{\mathbb{B}(v)} u(t) v(t) \leq \frac{1-v}{\mathbb{B}(v)} \frac{(u(t)+v(t))^{2}}{(1+\alpha)(\theta+1)} \leq \frac{1}{\alpha} \frac{1-v}{\mathbb{B}(v)} u(t) v(t), \\
& \frac{1}{\theta} \frac{v(t-s)^{v-1}}{\mathbb{B}(v) \Gamma(v)} u(t) v(t) \leq \frac{v(t-s)^{v-1}}{\mathbb{B}(v) \Gamma(v)} \frac{(u(t)+v(t))^{2}}{(1+\alpha)(\theta+1)} \leq \frac{1}{\alpha} \frac{v(t-s)^{v-1}}{\mathbb{B}(v) \Gamma(v)} u(t) v(t) .
\end{aligned}
$$

Integrating Eq. (3.53) from 0 to $t$ with respect to $s$, we have

$$
\frac{1}{\theta} \int_{a}^{t} \frac{v(t-s)^{v-1}}{\mathbb{B}(v) \Gamma(v)} u(s) v(s) d s \leq \int_{a}^{t} \frac{v(t-s)^{\nu-1}}{\mathbb{B}(v) \Gamma(v)} \frac{(u(s)+v(s))^{2}}{(1+\alpha)(\theta+1)} d s
$$




$$
\leq \frac{1}{\alpha} \int_{a}^{t} \frac{v(t-s)^{\nu-1}}{\mathbb{B}(\nu) \Gamma(v)} u(s) v(s) d s
$$

Adding Eqs. (3.52) and Eq. (3.54), we obtain the required inequality.

\title{
4 Conclusion
}

In this paper, we have considered Minkowski's inequality for the AB-fractional integral operator. We have also obtained some other types of integral inequalities for the ABfractional integral operator. By the help of this work we obtained more general inequalities than in the classical cases. For possible further work, we suggest to apply the obtained inequalities to prove the existence of solutions of fractional differential equations.

\begin{abstract}
Acknowledgements
The author Thabet Abdeljawad would like to thank Prince Sultan University for funding this work through research group Nonlinear Analysis Methods in Applied Mathematics (NAMAM), group number RG-DES-2017-01-17. All the authors are very grateful to the editorial board and the reviewers, whose comments improved the quality of the paper.
\end{abstract}

Funding

Not applicable.

Availability of data and materials

Data sharing not applicable to this paper as no datasets were generated during the current study.

Competing interests

The authors have no conflict of interests regarding the publication of this paper.

Authors' contributions

All the authors have equal contributions in this paper. All authors read and approved the final manuscript.

\section{Author details}

${ }^{1}$ College of Engineering Mechanics and Materials, Hohai University, Nanjing, P.R. China. ${ }^{2}$ Department of Mathematics, Shaheed BB University, Khybar Pakhtunkhwa, Pakistan. ${ }^{3}$ Department of Mathematics and General Sciences, Prince Sultan University, Riyadh, Saudi Arabia. ${ }^{4}$ Department of Mathematics, Faculty of Sciences, Van Yuzuncu Yil University, Van, Turkey. ${ }^{5}$ Department of Mathematics, College of Science, Hohai University, Nanjing, P.R. China. ${ }^{6}$ Department of Mathematics, University of Peshawar, Khybar Pakhtunkhwa, Pakistan.

\section{Publisher's Note}

Springer Nature remains neutral with regard to jurisdictional claims in published maps and institutional affiliations.

Received: 4 February 2019 Accepted: 27 March 2019 Published online: 08 April 2019

\section{References}

1. Baleanu, D., Khan, H., Jafari, H., Khan, R.A., Alipour, M.: On existence results for solutions of a coupled system of hybrid boundary value problems with hybrid conditions. Adv. Differ. Equ. 2015(1), 318 (2015)

2. Baleanu, D., Mustafa, O.G., Agarwal, P.R.: An existence result for a superlinear fractional differential equation. Appl. Math. Lett. 23(9), 1129-1132 (2010)

3. Baleanu, D., Mustafa, O.G., Agarwal, R.P.: On the solution set for a class of sequential fractional differential equations. J. Phys. A, Math. Theor. 43(38), 385209 (2010)

4. Baleanu, D., Agarwal, R.P., Khan, H., Khan, R.A., Jafari, H.: On the existence of solution for fractional differential equations of order $3<\delta<4$. Adv. Differ. Equ. 2015, 362 (2015)

5. Baleanu, D., Agarwal, R.P., Mohammadi, H., Rezapour, S.: Some existence results for a nonlinear fractional differential equation on partially ordered Banach spaces. Bound. Value Probl. 2013, 112 (2013)

6. Agarwal, P., Jleli, M., Tomar, M.: Certain Hermite-Hadamard type inequalities via generalized $k$-fractional integrals. J. Inequal. Appl. 2017(1), 55 (2017)

7. Aldhaifallah, M., Tomar, M., Nisar, K.S., Purohit, S.D.: Some new inequalities for (k, s)-fractional integrals. J. Nonlinear Sci. Appl. 9, 5374-5381 (2016)

8. Set, E., Noor, M.A., Awan, M.U., Gzpinar, A.: Generalized Hermite-Hadamard type inequalities involving fractional integral operators. J. Inequal. Appl. 2017, 169 (2017)

9. Khan, H., Tunç, C., Alkhazan, A., Ameen, B., Khan, A.: A generalization of Minkowski's inequality by Hahn integral operator. J. Taibah Univ. Sci. 12(5), 506-513 (2018)

10. Baleanu, D., Fernandez, A.: On some new properties of fractional derivatives with Mittag-Leffler kernel. Commun. Nonlinear Sci. Numer. Simul. 59, 444-462 (2018)

11. Abdeljawad, T., Baleanu, D.: Integration by parts and its applications of a new nonlocal fractional derivative with Mittag-Leffler nonsingular kernel. J. Nonlinear Sci. Appl. 10(3), 1098-1107 (2017) 
12. Abdeljawad, T.: A Lyapunov type inequality for fractional operators with nonsingular Mittag-Leffler kernel. J. Inequal. Appl. 2017, 130 (2017). https://doi.org/10.1186/s13660-017-1400-5

13. Abdeljawad, T., Baleanu, D:: On fractional derivatives with generalized Mittag-Leffler kernels. Adv. Differ. Equ. 2018 , $468(2018)$

14. Abdeljawad, T.: Fractional operators with generalized Mittag-Leffler kernels and their iterated differintegrals. Chaos 29, 023102 (2019). https://doi.org/10.1063/1.5085726

15. Khan, H., Tunç, C., Baleanu, D., Khan, A., Alkhazzan, A.: Inequalities for $n$-class of functions using the Saigo fractional integral operator. Rev. R. Acad. Cienc. Exactas Fís. Nat., Ser. A Mat. 1-4 (2019, to appear). https://doi.org/10.1007/s13398-019-00624-5

16. Jarad, F., Abdeljawad, T., Hammouch, Z:: On a class of ordinary differential equations in the frame of Atangana-Baleanu fractional derivative. Chaos Solitons Fractals 117, 16-20 (2021)

17. Adjabi, Y., Jarad, F., Abdeljawad, T.: On generalized fractional operators and a Gronwall type inequality with applications. Filomat 31(17), 5457-5473 (2017)

18. Shuang, Y., Qi, F.: Integral inequalities of Hermite-Hadamard type for extended s-convex functions and applications. Mathematics 6(11), 223 (2018)

19. Mehrez, K., Agarwal, P.: New Hermite-Hadamard type integral inequalities for convex functions and their applications. J. Comput. Appl. Math. 350, 274-285 (2019)

20. Park, M.J., Kwon, O.M., Ryu, J.H.: Generalized integral inequality: application to time-delay systems. Appl. Math. Lett. 77, 6-12 (2018)

21. Sarikaya, M.Z., Tunc, T., Budak, H.: On generalized some integral inequalities for local fractional integrals. Appl. Math. Comput. 276, 316-323 (2016)

22. Farid, G., Khan, K.A., Latif, N., Rehman, A.U., Mehmood, S.: General fractional integral inequalities for convex and m-convex functions via an extended generalized Mittag-Leffler function. J. Inequal. Appl. 2018, 243 (2018)

23. Sarikaya, M.Z., Set, E., Yaldiz, H., Basak, N.: Hermite-Hadamard's inequality for fractional integrals and related fractional inequalities. Math. Comput. Model. 57, 2403-2407 (2013)

24. Mohammed, P.O.: On new trapezoid type inequalities for $h$-convex functions via generalized fractional integrals. Turk J. Anal. Number Theory 6, 125-128 (2018)

25. Mohammed, P.O., Sarikaya, M.Z.: Hermite-Hadamard type inequalities for F-convex function involving fractional integrals. J. Inequal. Appl. 2018, 359 (2018)

26. Jafari, H., Jassim, H.K., Moshokoa, S.P., Ariyan, V.M., Tchier, F.: Reduced differential transform method for partial differential equations within local fractional derivative operators. Adv. Mech. Eng. 8(4), 1-6 (2016)

27. Richard, H.: Fractional Calculus: An Introduction for Physicists. World Scientific, Singapore (2014)

28. Podlubny, I.: Fractional Differential Equations: An Introduction to Fractional Derivatives, Fractional Differential Equations, to Methods of Their Solution and Some of Their Applications. Elsevier, Amsterdam (1998)

29. Sun, H., Zhang, Y., Chen, W., Reeves, D.M.: Use of a variable-index fractional-derivative model to capture transient dispersion in heterogeneous media. J. Contam. Hydrol. 157, 47-58 (2014)

30. Owolabi, K.M.: Modelling and simulation of a dynamical system with the Atangana-Baleanu fractional derivative. Eur. Phys. J. Plus 133(1), 15 (2018)

31. Kumar, D., Singh, J., Baleanu, D.: Analysis of regularized long-wave equation associated with a new fractional operator with Mittag-Leffler type kernel. Phys. A, Stat. Mech. Appl. 492, 155-167 (2018)

32. Jianke, Z., Gaofeng, W., Xiaobin, Z., Chang, Z.: Generalized Euler-Lagrange equations for fuzzy fractional variational problems under gH-Atangana-Baleanu differentiability. Hindawi 2018, Article ID 2740678 (2018). https://doi.org/10.1155/2018/2740678

33. Abdeljawad, T., Baleanu, D.: Discrete fractional differences with nonsingular discrete Mittag-Leffler kernels. Adv. Differ. Equ. 2016, 232 (2016). https://doi.org/10.1186/s13662-016-0949-5

\section{Submit your manuscript to a SpringerOpen ${ }^{\circ}$ journal and benefit from:}

- Convenient online submission

- Rigorous peer review

- Open access: articles freely available online

- High visibility within the field

Retaining the copyright to your article

Submit your next manuscript at $\gg$ springeropen.com 